\title{
Application of interval-valued intuitionistic fuzzy sets of second type in pattern recognition
}

\author{
K. Rajesh and R. Srinivasan \\ Department of Mathematics, Islamiah College (Autonomous) \\ Vaniyambadi-635752, India \\ e-mails: rajeshagmegmail.com, \\ srinivasanmaths@yahoo.com
}

Received: 22 October 2017 Revised: 28 November $2017 \quad$ Accepted: 1 December 2017

\begin{abstract}
After the introduction of intuitionistic fuzzy sets (IFS), many researchers have confirmed the resourcefulness of IFSs in decision making problems like pattern recognition, machine learning, medical diagnosis, electoral system, career determination, market prediction, and so on. In this paper, we propose the new distance measures on interval-valued Intuitionistic Fuzzy Sets of Second Type and the application of interval-valued Intuitionistic Fuzzy Sets of Second Type in Pattern Recognition by using normalized hamming distance measure and compare the result with the existing IVIFS.
\end{abstract}

Keywords: Intuitionistic fuzzy sets, Intuitionistic fuzzy sets of second type, Interval-valued intuitionistic fuzzy sets, Interval-valued intuitionistic fuzzy sets of second type.

2010 Mathematics Classification: 03B20, 03D45, 03E72, 03F55.

\section{Introduction}

An Intuitionistic Fuzzy Set (IFS) for a given underlying set $X$ was introduced by K. T. Atanassov [1] as a generalization of ordinary Fuzzy Sets (FS, see [9]), and he introduced the theory of Interval-Valued Intuitionistic Fuzzy Sets (IVIFS) and established some of their properties. Many researchers have contributed their research work in the application of IVIFS in pattern recognition. Most of the applications of IFSs and their extensions are carried out using distance measures approach $[4,5]$. Distance measure between intuitionistic fuzzy sets is an important concept in 
fuzzy mathematics because of its wide applications in real life situations like pattern recognition, machine learning, medical diagnosis, electoral system, career determination, market prediction, and so on as in $[7,8]$. In this paper we present the application of IVIFSST in Pattern Recognition by using normalized hamming distance measure and compare the result with the existing IVIFS.

The rest of the paper is designed as follows: In Section 2, we give some basic definitions. In Section 3, we propose the various distance measures on interval-valued intuitionistic fuzzy sets of second type with suitable examples. In Section 4, we give the application of IVIFSST in pattern recognition and analyzed the results. This paper is concluded in Section 5.

\section{Preliminaries}

In this section, we give some basic definitions.

Definition 2.1. [3] Let X be a non-empty set. An Intuitionistic Fuzzy Set (IFS) A in X is defined as an object of the following form

$$
A=\left\{\left\langle x, \mu_{A}(x), \nu_{A}(x)\right\rangle \mid x \in X\right\},
$$

where the functions $\mu_{A}: X \rightarrow[0,1]$ and $\nu_{A}: X \rightarrow[0,1]$ denote the degree of membership and the degree of non-membership of the element $x \in X$, respectively, and for every $x \in X$ :

$$
0 \leq \mu_{A}(x)+\nu_{A}(x) \leq 1
$$

Definition 2.2. [3] An Intuitionistic Fuzzy Sets of Second Type (IFSST) A in X is defined as an object of the following form

$$
A=\left\{\left\langle x, \mu_{A}(x), \nu_{A}(x)\right\rangle \mid x \in X\right\},
$$

where the functions $\mu_{A}: X \rightarrow[0,1]$ and $\nu_{A}: X \rightarrow[0,1]$ denote the degree of membership and the degree of non-membership of the element $x \in X$, respectively, and for every $x \in X$ :

$$
0 \leq \mu_{A}^{2}(x)+\nu_{A}^{2}(x) \leq 1 .
$$

Definition 2.3. [2] Let $X$ be an universal set with cardinality $n$. Let $[0,1]$ be the set of all closed subintervals of the interval $[0,1]$ and elements of this set are denoted by uppercase letters. If $M \in[0,1]$ then it can be represented as $M=\left[M_{L}, M_{U}\right]$, where $M_{L}$ and $M_{U}$ are the lower and upper limits of $M$. For $M \in[0,1], \bar{M}=1-M$ represents the interval $\left[1-M_{L}, 1-M_{U}\right]$ and $W_{M}=M_{U}-M_{L}$ is the width of $M$.

An Interval-Valued Fuzzy Set (IVFS) A in X is given by

$$
A=\left\{\left\langle x, M_{A}(x)\right\rangle \mid x \in X\right\},
$$

where $M_{A}: X \rightarrow[0,1], M_{A}(x)$ denote the degree of membership of the element $x$ to the set $A$. 
Definition 2.4. [2] An Interval-Valued Intuitionistic Fuzzy Set (IVIFS) A in X is given by

$$
A=\left\{\left\langle x, M_{A}(x), N_{A}(x)\right\rangle \mid x \in X\right\}
$$

where $M_{A}: X \rightarrow[0,1], N_{A}: X \rightarrow[0,1]$. The intervals $M_{A}(x)$ and $N_{A}(x)$ denote the degree of membership and the degree of non-membership of the element $x$ to the set $A$, where $M_{A}(x)=$ $\left[M_{A L}(x), M_{A U}(x)\right]$ and $N_{A}(x)=\left[N_{A L}(x), N_{A U}(x)\right]$ with the condition that

$$
M_{A U}(x)+N_{A U}(x) \leq 1 \text { for all } x \in X,
$$

Definition 2.5. [6] An Interval-Valued Intuitionistic Fuzzy Sets of Second Type (IVIFSST) A in X is given by

$$
A=\left\{\left\langle x, M_{A}(x), N_{A}(x)\right\rangle \mid x \in X\right\},
$$

where $M_{A}: X \rightarrow[0,1], N_{A}: X \rightarrow[0,1]$. The intervals $M_{A}(x)$ and $N_{A}(x)$ denote the degree of membership and the degree of non-membership of the element $x$ to the set $A$, where $M_{A}(x)=$ $\left[M_{A L}(x), M_{A U}(x)\right]$ and $N_{A}(x)=\left[N_{A L}(x), N_{A U}(x)\right]$ with the condition that

$$
M_{A U}^{2}(x)+N_{A U}^{2}(x) \leq 1 \text { for all } x \in X
$$

Definition 2.6. [7] Let $X$ be nonempty such that IVIFS A, B, C $\in X$. Then the distance measure between IVIFS $A$ and $B$ is a mapping $d: X \times X \rightarrow[0,1]$; if $d(A, B)$ satisfies the following axioms:

$$
\begin{aligned}
& A 10 \leq d(A, B) \leq 1 \\
& A 2 d(A, B)=0 \text { if and only if } A=B \\
& A 3 d(A, B)=d(B, A) \\
& A 4 d(A, C)+d(B, C) \geq d(A, B) ; \\
& A 5 \text { if } A \subseteq B \subseteq C, \text { then } d(A, C) \geq d(A, B) \text { and } d(A, C) \geq d(B, C)
\end{aligned}
$$

Definition 2.7. [3] Let $A$ and $B$ be two IVIFSs in $X=\left\{x_{1}, x_{2}, \ldots, x_{n}\right\}$, then the various distance measures are defined as follows.

Hamming Distance between $\boldsymbol{A}$ and $B$ is $d_{H I V I F S}(A, B)$

$$
\begin{array}{r}
=\frac{1}{4} \sum_{i=1}^{n}\left|M_{A L}\left(x_{i}\right)-M_{B L}\left(x_{i}\right)\right|+\left|M_{A U}\left(x_{i}\right)-M_{B U}\left(x_{i}\right)\right|+\left|N_{A L}\left(x_{i}\right)-N_{B L}\left(x_{i}\right)\right|+ \\
\left|N_{A U}\left(x_{i}\right)-N_{B U}\left(x_{i}\right)\right|+\left|\pi_{A L}\left(x_{i}\right)-\pi_{B L}\left(x_{i}\right)\right|+\left|\pi_{A U}\left(x_{i}\right)-\pi_{B U}\left(x_{i}\right)\right|
\end{array}
$$

Normalized Hamming Distance between $A$ and $B$ is

$$
d_{H I V I F S}^{\prime}(A, B)=\frac{d_{H I V I F S}(A, B)}{n}
$$


Euclidean Distance between $\boldsymbol{A}$ and $\boldsymbol{B}$ is $d_{E I V I F S}(A, B)$

$$
\begin{array}{r}
=\frac{1}{2} \sum_{i=1}^{n}\left[\left(M_{A L}\left(x_{i}\right)-M_{B L}\left(x_{i}\right)\right)^{2}+\left(M_{A U}\left(x_{i}\right)-M_{B U}\left(x_{i}\right)\right)^{2}+\left(N_{A L}\left(x_{i}\right)-N_{B L}\left(x_{i}\right)\right)^{2}+\right. \\
\left.\left(N_{A U}\left(x_{i}\right)-N_{B U}\left(x_{i}\right)\right)^{2}+\left(\pi_{A L}\left(x_{i}\right)-\pi_{B L}\left(x_{i}\right)\right)^{2}+\left(\pi_{A U}\left(x_{i}\right)-\pi_{B U}\left(x_{i}\right)\right)^{2}\right]^{0.5}
\end{array}
$$

\section{Normalized Euclidean Distance between $A$ and $B$ is}

$$
d_{E I V I F S}^{\prime}(A, B)=\frac{d_{E I V I F S}(A, B)}{\sqrt{n}}
$$

Example 2.1. Let us consider two Interval-valued Intuitionistic Fuzzy Sets $A$ and $B$ in $X=$ $\{a, b, c, d\}$ as follows:

$$
\begin{aligned}
A & =\{\langle[0.6,0.3],[0.2,0.2],[0.2,0.5]\rangle,\langle[0.5,0.3],[0.3,0.4],[0.2,0.3]\rangle\} \\
B & =\{\langle[0.5,0.2],[0.4,0.3],[0.1,0.5]\rangle,\langle[0.4,0.3],[0.1,0.3],[0.5,0.4]\rangle\}
\end{aligned}
$$

Then,

(i) The Hamming distance between $A$ and $B$ is equal to 0.23.

(ii) The Normalized Hamming distance between $A$ and $B$ is equal to 0.12.

(iii) The Euclidean distance between $A$ and $B$ is equal to 0.18 .

(iv) The Normalised Euclidean distance between $A$ and $B$ is equal to 0.13.

\section{Some distance measures over IVIFSST}

In this section, we introduce the various distance measures on IVIFSST with example.

Definition 3.1. Let $A$ and $B$ be two IVIFSSTs in $X=\left\{x_{1}, x_{2}, \ldots, x_{n}\right\}$, then the various distance measures are defined as follows.

Hamming Distance between $\boldsymbol{A}$ and $\boldsymbol{B}$ is $d_{H I V I F S S T}(A, B)$

$$
\begin{aligned}
=\frac{1}{4} \sum_{i=1}^{n}\left[\mid M_{A L}^{2}\left(x_{i}\right)-\right. & M_{B L}^{2}\left(x_{i}\right)|+| M_{A U}^{2}\left(x_{i}\right)-M_{B U}^{2}\left(x_{i}\right)|+| N_{A L}^{2}\left(x_{i}\right)-N_{B L}^{2}\left(x_{i}\right) \mid+ \\
& \left.\left|N_{A U}^{2}\left(x_{i}\right)-N_{B U}^{2}\left(x_{i}\right)\right|+\left|\pi_{A L}^{2}\left(x_{i}\right)-\pi_{B L}^{2}\left(x_{i}\right)\right|+\left|\pi_{A U}^{2}\left(x_{i}\right)-\pi_{B U}^{2}\left(x_{i}\right)\right|\right]
\end{aligned}
$$

Normalized Hamming Distance between A and B is

$$
d_{H I V I F S S T}^{\prime}(A, B)=\frac{d_{H I V I F S S T}(A, B)}{n}
$$

Euclidean Distance between A and B is $d_{E I V I F S S T}(A, B)$

$$
\begin{aligned}
&=\frac{1}{2} \sum_{i=1}^{n}\left[\left(M_{A L}^{2}\left(x_{i}\right)-\right.\right.\left.M_{B L}^{2}\left(x_{i}\right)\right)^{2}+\left(M_{A U}^{2}\left(x_{i}\right)-M_{B U}^{2}\left(x_{i}\right)\right)^{2}+\left(N_{A L}^{2}\left(x_{i}\right)-N_{B L}^{2}\left(x_{i}\right)\right)^{2}+ \\
&\left.\left(N_{A U}^{2}\left(x_{i}\right)-N_{B U}^{2}\left(x_{i}\right)\right)^{2}+\left(\pi_{A L}^{2}\left(x_{i}\right)-\pi_{B L}^{2}\left(x_{i}\right)\right)^{2}+\left(\pi_{A U}^{2}\left(x_{i}\right)-\pi_{B U}^{2}\left(x_{i}\right)\right)^{2}\right]^{\frac{1}{2}}
\end{aligned}
$$

Normalized Euclidean Distance between $A$ and $B$ is

$$
d_{\text {EIVIFSST }}^{\prime} A, B=\frac{d_{E I V I F S S T}(A, B)}{\sqrt{n}} .
$$


Example 3.1. Let us consider two Interval-valued Intuitionistic Fuzzy Sets of Second Type A and $\mathrm{B}$ in $X=\{a, b, c, d\}$ as follows:

$$
\begin{aligned}
& A=\{\langle[0.6,0.3],[0.2,0.2],[0.2,0.5]\rangle,\langle[0.5,0.3],[0.3,0.4],[0.2,0.3]\rangle\} \\
& B=\{\langle[0.5,0.2],[0.4,0.3],[0.1,0.5]\rangle,\langle[0.4,0.3],[0.1,0.3],[0.5,0.4]\rangle\}
\end{aligned}
$$

Then,

(i) The Hamming distance between $A$ and $B$ is equal to 0.15

(ii) The Normalised Hamming distance between $A$ and $B$ is equal to 0.08

(iii) The Euclidean distance between $A$ and $B$ is equal to 0.37

(iv) The Normalised Euclidean distance between $A$ and $B$ is equal to 0.26

From the above results, we infer that the normalized Hamming distance gives the best distance measure between $A$ and $B$. This is because the distance is the shortest or smallest. For this reason, we shall make use of normalized hamming distance in the applications for its high rate of confidence in terms of accuracy.

\section{Application of IVIFSST in pattern recognition}

In this process, a sets of patterns and another unknown pattern called sample is given (IVIFSST in nature). Both the set of the pattern and that of the sample are within the same feature space or attributes $n$. The aim is to find the distance between each of the patterns and the sample. The smallest or shortest distance between any of the patterns and the sample shows that, the sample belongs to that pattern. Assume that there exist $n$ patterns given by,

$$
A_{j}=\left\{\left\langle x_{i},\left[M_{A L}\left(x_{i}\right), M_{A U}\left(x_{i}\right)\right],\left[N_{A L}\left(x_{i}\right), N_{A U}\left(x_{i}\right)\right]\right\rangle \mid x \in X\right\}, i=1,2, \ldots n
$$

and $A_{j}=\left\{A_{1}, A_{2}, A_{3}, \ldots . A_{m}\right\}$ for $m \in N$. Suppose that there is a sample to be recognized, that is

$$
B_{j}=\left\{\left\langle x_{i},\left[M_{B L}\left(x_{i}\right), M_{B U}\left(x_{i}\right)\right],\left[N_{B L}\left(x_{i}\right), N_{B U}\left(x_{i}\right)\right]\right\rangle \mid x \in X\right\}, \text { for } i=1,2, \ldots n .
$$

For example, let six patterns be represented by IVIFSSTs in $X=\left\{x_{1}, x_{2}, x_{3}, x_{4}\right\}$ (i.e. feature space for $n=4)$ as

$$
\begin{gathered}
A_{1}=\{\langle[0.5,0.3],[0.2,0.6],[0.3,0.1]\rangle,\langle[0.3,0.8],[0.5,0.1],[0.2,0.1]\rangle, \\
\langle[0.7,0.4],[0.1,0.5],[0.2,0.1]\rangle,\langle[0.6,0.3],[0.4,0.4],[0.0,0.3]\rangle\} \\
A_{2}=\{\langle[0.4,0.5],[0.3,0.4],[0.3,0.1]\rangle,\langle[0.1,0.3],[0.5,0.2],[0.4,0.5]\rangle, \\
\langle[0.5,0.4],[0.3,0.3],[0.2,0.3]\rangle,\langle[0.7,0.4],[0.0,0.5],[0.3,0.1]\rangle\} \\
A_{3}=\{\langle[0.8,0.1],[0.1,0.6],[0.1,0.3]\rangle,\langle[0.4,0.6],[0.2,0.2],[0.4,0.2]\rangle, \\
\langle[0.5,0.4],[0.4,0.3],[0.1,0.3]\rangle,\langle[0.1,0.8],[0.5,0.2],[0.4,0.0]\rangle\} \\
A_{4}=\{\langle[0.5,0.4],[0.2,0.3],[0.3,0.3]\rangle,\langle[0.2,0.4],[0.3,0.5],[0.5,0.1]\rangle, \\
\langle[0.7,0.2],[0.3,0.0],[0.0,0.8]\rangle,\langle[0.4,0.3],[0.2,0.1],[0.4,0.6]\rangle\} \\
A_{5}=\{\langle[0.2,0.3],[0.4,0.4],[0.4,0.3]\rangle,\langle[0.7,0.2],[0.1,0.7],[0.2,0.1]\rangle, \\
\langle[0.0,0.3],[0.4,0.4],[0.6,0.3]\rangle,\langle[0.3,0.3],[0.4,0.6],[0.3,0.1]\rangle\} \text { and } \\
A_{6}=\{\langle[0.1,0.2],[0.3,0.4],[0.6,0.4]\rangle,\langle[0.5,0.4],[0.4,0.5],[0.1,0.1]\rangle, \\
\langle[0.3,0.4],[0.5,0.3],[0.2,0.3]\rangle,\langle[0.7,0.8],[0.0,0.2],[0.3,0.0]\rangle\}
\end{gathered}
$$

be the classification of building materials. 
Consider another kind of unknown building material $B$ as $B=\{\langle[0.6,0.3],[0.3,0.4],[0.1,0.3]\rangle,\langle[0.5,0.4],[0.5,0.4],[0.0,0.2]\rangle$,

$$
\langle[0.3,0.2],[0.6,0.7],[0.1,0.1]\rangle,\langle[0.4,0.3],[0.1,0.4],[0.5,0.3]\rangle\}
$$

for all $A, B \in X$ Our task is to show which class of $A_{j}$ (for $j=1,2,3,4,5,6$ ), the unknown pattern belongs to.

\subsection{Results and discussion}

Using normalized hamming distance measure for IVIFS, we have the following results:

$d_{H I V I F S}^{\prime}\left(A_{1}, B\right)=0.5500, d_{H I V I F S}^{\prime}\left(A_{2}, B\right)=0.5750, d_{H I V I F S}^{\prime}\left(A_{3}, B\right)=\mathbf{0 . 6 2 5 0}$,

$d_{H I V I F S}^{\prime}\left(A_{4}, B\right)=0.6000, d_{H I V I F S}^{\prime}\left(A_{5}, B\right)=0.6000$ and $d_{H I V I F S}^{\prime}\left(A_{6}, B\right)=\mathbf{0 . 5 2 5 0}$.

From these results, we see that, the distance between $A_{6}$ and $B$ is the smallest, and the distance between $A_{3}$ and $B$ is the greatest. Since $A_{6}$ approaches $B$, we say that the unknown pattern $B$ belongs to $A_{6}$.

Again using normalized hamming distance measure for IVIFSST we have the following results:

$d_{H I V I F S S T}^{\prime}\left(A_{1}, B\right)=0.3887, d_{H I V I F S S T}^{\prime}\left(A_{2}, B\right)=0.3900, d_{H I V I F S S T}^{\prime}\left(A_{3}, B\right)=\mathbf{0 . 4 5 2 5}$

$d_{\text {HIVIFSST }}^{\prime}\left(A_{4}, B\right)=0.4412, d_{\text {HIVIFSST }}^{\prime}\left(A_{5}, B\right)=0.4125$ and $d_{H I V I F S S T}^{\prime}\left(A_{6}, B\right)=\mathbf{0 . 3 8 0 0}$.

From these results, we see that, in both the cases the distance between $A_{6}$ and $B$ is the smallest, and the distance between $A_{3}$ and $B$ is the greatest since $A_{6}$ approaches $B$, we say that the unknown pattern $B$ belongs to $A_{6}$.

We conclude that, IVIFSST plays a vital role in obtaining the shortest distance when compared with IVIFS.

\section{Conclusion}

In this paper, we have introduced the various distance measures on IVIFSST in particular, we have applied the normalized hamming distance measure on IVIFSST for calculating the shortest distance. Also we have compared the results with existing IVIFS. It is observed that IVIFSST is the good tool for finding the shortest distance.

\section{References}

[1] Atanassov, K. T. (1986) Intuitionistic fuzzy sets, Fuzzy Sets and Systems, 20(1), 87-96.

[2] Atanassov, K. T. \& Gargov, G. (1989) Interval-Valued Fuzzy Sets, Fuzzy Sets and Systems, 31, 343-349.

[3] Atanassov, K. T. (1999) Intuitionistic Fuzzy Sets: Theory and Applications, Springer Verlag, Heidelberg. 
[4] Ejegwa, P. A., Onoja, A. M., \& Emmanuel, I. T. (2010) A Note on Some Models of Intuitionistic Fuzzy Sets in Real Life Situations, Journal of Global Research in Mathematical Archives, 2(5), 42-50.

[5] Ejegwa, P. A., Akubo, A. J. \& Joshua, O. M. (2014) Intuitionistic fuzzy set and its application in career determination via normalized Euclidean distance method, European Scientific Journal, 10(15), 529-536.

[6] Rajesh, K., \& Srinivasan, R. (2017) Interval-valued Intuitionistic Fuzzy Sets of Second Type, Advances in Fuzzy Mathematics, 12(4), 845-853.

[7] Szmidt, E., \& Kacprzyk, J. (2001) Intuitionistic fuzzy sets in intelligent data analysis for medical diagnostic, Lecture Notes in Computer Science, 2074, 263-271.

[8] Szmidt, E., \& Kacprzyk, J. (2001) Intuitionistic fuzzy sets in some medical applications, Note on Intuitionistic Fuzzy Sets, 7(4), 58-64.

[9] Zadeh, L. A. (1965) Fuzzy sets, Information and Control, 8, 338-353. 